\title{
3D Nano-Fabrication Using Controlled Bessel-Glass Interaction in Ultra- fast Modes
}

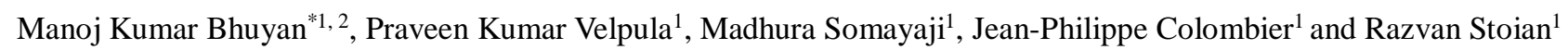 \\ ${ }^{* 1}$ Laboratoire Hubert Curien, UMR 5516 CNRS, Université de Lyon, Université Jean Monnet, \\ 42000 Saint Etienne, France \\ E-mail: manoj.femto@gmail.com \\ 2 Presently at RIKEN-SIOM Joint Research Unit, RIKEN Center for Advanced Photonics, 2-1 Hi- \\ rosawa, Wako, Saitama 351-0198, Japan
}

\begin{abstract}
With a quest to develop rapid and reliable three-dimensional nanofabrication techniques for photonic and fluidic applications, we exploited the potential of zero-order Bessel beams for fabricating high aspect ratio nano-structures in fused silica glass. In particular, the energetic conditions towards stable and uniform nanovoid fabrication were explored, correlating the laser pulse energy and temporal duration. The underlying carrier and structural dynamics revealed through multiscale timeresolved microscopy techniques in the case of Bessel pulse excitation of silica glass were also studied. We discovered different material relaxation pathways corresponding to material modifications of different morphologies such as increased and decreased refractive index structures.
\end{abstract}

DOI: 10.2961/jlmn.2017.03.0017

Keywords: nanofabrication, picosecond laser, Bessel beams, ultrafast phenomena, silica glass

\section{Introduction}

In general, the progress of science and technology at nanoscale urges rapid and reliable 3-dimensional (3D) nanoscale fabrication techniques, especially considering 3D photonic and fluidic applications. Deep drilling capability of nanoscale machining techniques is thus become more relevant. For example, to achieve photonic bandgaps at visible spectral range, $3 \mathrm{D}$ photonic crystals comprising of nanochannel- array of sub-200 nm lateral size and few tens of microns long uniform channels, would be required. Traditional methodologies in combination with contemporary approaches involving lithography and plasma etching (essentially planar), attaining sub-100 nm fabrication resolution, are unable to address the deep drilling issue. The state-of-the-art electron/ion beam based machining techniques are capable to machine $3 \mathrm{D}$ nanochannels (must be connected to one of the sample surfaces) but up to a few microns deep, and the respective machined channels also suffer from taper characteristics.

In this context, laser based material processing techniques are shown to modify materials in $3 \mathrm{D}$ and with a spatial resolution down to few tens of nanometers [1-4]. Note that although nanoscale structures can be fabricated in transparent materials using ultrashort laser pulses of standard Gaussian spatial profiles, and exploiting tight focusing geometries, long yet narrow (i.e. sub-micron scale) structure fabrication is limited due to the inherent focal spot characteristics i.e. short Rayleigh range. Spherical aberration and nonlinear beam propagation effects [5, 6] were explored to increase the longitudinal extent of fabricated structure and indeed nanochannels of diameters down to $200 \mathrm{~nm}$ and lengths up to few microns were obtained in glass.

The beam engineering techniques have shown great potential in tackling the above-mentioned issue. For instance, spatially engineered laser beams like non-diffractive zeroorder Bessel beams [7, 8], with a very narrow central core extended over long distance, are demonstrated to be beneficial in machining high aspect ratio (length / width) nanostructures on surface as well as in bulk transparent materials [9-16]. Pulse dispersion engineering and timeshaping can equally assist in controlling the light-matter interaction processes [14]. In this study, we further exploit the potential of zero-order Bessel beams for obtaining long, uniform material modifications, such as increased refractive index (Type I) and decreased refractive index (Type II) structures in silica glass on single-shot basis. The fundamental mechanisms of such nanoscale modified structures in this unique light-matter interaction geometry were also subjects of the present study. The time-resolved measurements were carried out over a time window ranging from few hundred femtoseconds to few tens of microseconds to uncover the corresponding carrier and structural dynamics.

\section{Experimental arrangements and procedures}

In this section, the experimental arrangements and procedures that were followed for this study are described. In particular, the following topics are discussed. (i) How highquality zero-order Bessel beams were generated? (ii) How the laser machining experiments were performed? (iii) How Bessel beam induced material excitation and relaxation processes were studied?

\subsection{High-quality Bessel beam generation}

The experiments of this study employed ultrafast laser pulses emitting from a Ti:Sapphire laser system (central wavelength of $800 \mathrm{~nm}$, full width at half maxima (FWHM) pulse duration of 50 femtoseconds and $1 \mathrm{kHz}$ repetition rate). The laser beams of Gaussian spatial profile delivered from the above-mentioned laser system were further 
shaped into zero-order Bessel beams using an axicon lens [17] (Thorlabs Inc., AX2505-B model, base angle, $\alpha$ of $0.5^{\circ}$, UVFS glass of refractive index, $\mathrm{n}_{\mathrm{ax}}$ of 1.45). The Bessel beams were thus formed by the conical intersection of wavefronts refracted by the axicon. Here, two primary issues normally arise when such axicon generated Bessel beams are directly employed for machining applications. First, the Bessel beams that are generated using axicon lens start right at the tip of the axicon lens and therefore this methodology imposes constraints especially on bulk structuring applications where Bessel beams need to be formed inside the sample. The issue is even more serious when applications requiring high angle Bessel beams are targeted. Therefore, high angle Bessel beams are normally produced using axicon lens followed by a telescopic system. In the present case, the telescope comprises of two lenses: Planoconvex lens L1 (f1 = $1000 \mathrm{~mm}$ ) and lens L2 (Mitutoyo objective lenses of two different focal lengths, $\mathrm{f} 2=10$ and $20 \mathrm{~mm}$ depending on the requirement) as illustrated in Fig. 1(a). The final Bessel beam was thus produced with an onset position at the focal plane of lens L2.

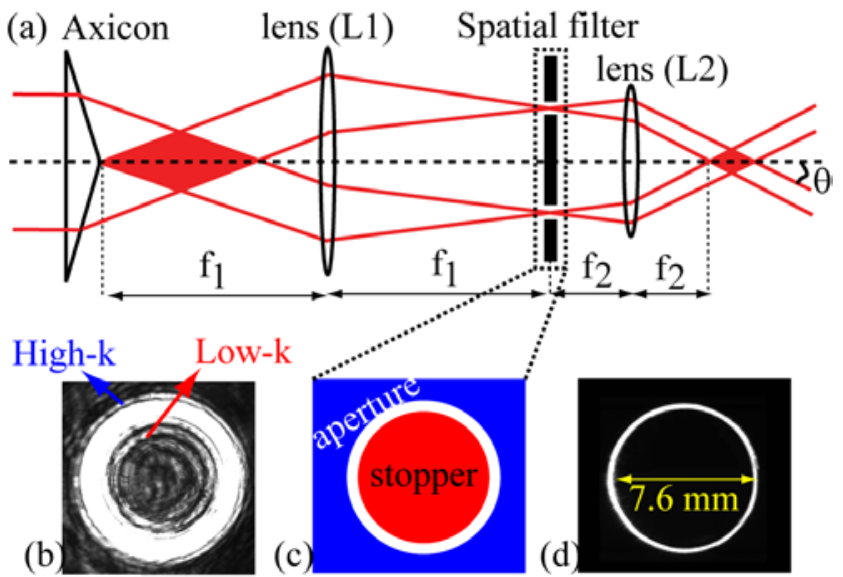

Fig. 1 Zero-order Bessel beam generation and filtering. (a) Experimental arrangement for high-quality Bessel beam generation using an axicon lens (base angle of $0.5^{\circ}$ ) followed by a telescopic system comprising of two lenses ( $\mathrm{f} 1=1000 \mathrm{~mm}$ and $\mathrm{f} 2=10$ and $20 \mathrm{~mm}$ ). (b) Saturated image of the ring pattern recorded at the focal plane of lens (L1), showing the undesired high and low wave vectors $(\mathrm{k})$ originated from surface quality and morphology of axicon lens. (c) Diagram of an ideal filtering system comprising of a stopper and an aperture. (d) Image of a filtered ring pattern at the focal plane of lens L1, that upon Fourier transformation leads to a high quality Bessel beam.

The second issue relates to the beam uniformity i.e. imperfect tip of axicon lens can result in an undesired axial intensity modulation of generated Bessel beams primarily due to the leakage of Gaussian components. This intensity modulation can leave signatures in the material modifications when using such Bessel beams. Therefore, efforts were made to produce high-quality Bessel beams using filtering systems. To do so, earlier study [18] proposed to use a stopper at the Fourier plane of lens L1 to block unwanted low spatial frequency components. However, we experimentally realized that it is also important to remove the undesired high spatial frequency components those are generated either from the axicon lens and/or from the light scattering from involved optical components (see Fig. 1(b)).
Therefore, we propose to use a novel "spatial filter" comprising of a stopper and an aperture (see Fig. 1(c)) and indeed experimentally generated a high-quality Bessel beam. By choosing the right sizes of stopper and aperture of the spatial filter, the spatial frequency components corresponding to a near-ideal Bessel beam were selected, by allowing them to pass through its annular opening (see Fig. 1(c)). The width of the annular opening was optimized to few hundred microns on the basis of the uniformity of axial intensity profile of the filtered Bessel beam. From a technical viewpoint, the aperture was a commercial mechanical aperture, with a possibility to set its opening diameter (effectively decides the outer diameter of annular ring). The stopper was prepared by fixing a thick opaque disk of paper of suitable diameter (effectively decides the inner diameter of annular ring) on a $150 \mu \mathrm{m}$ thick glass microscope cover slide $\left(18 \times 18 \mathrm{~mm}^{2}\right)$.

(a)
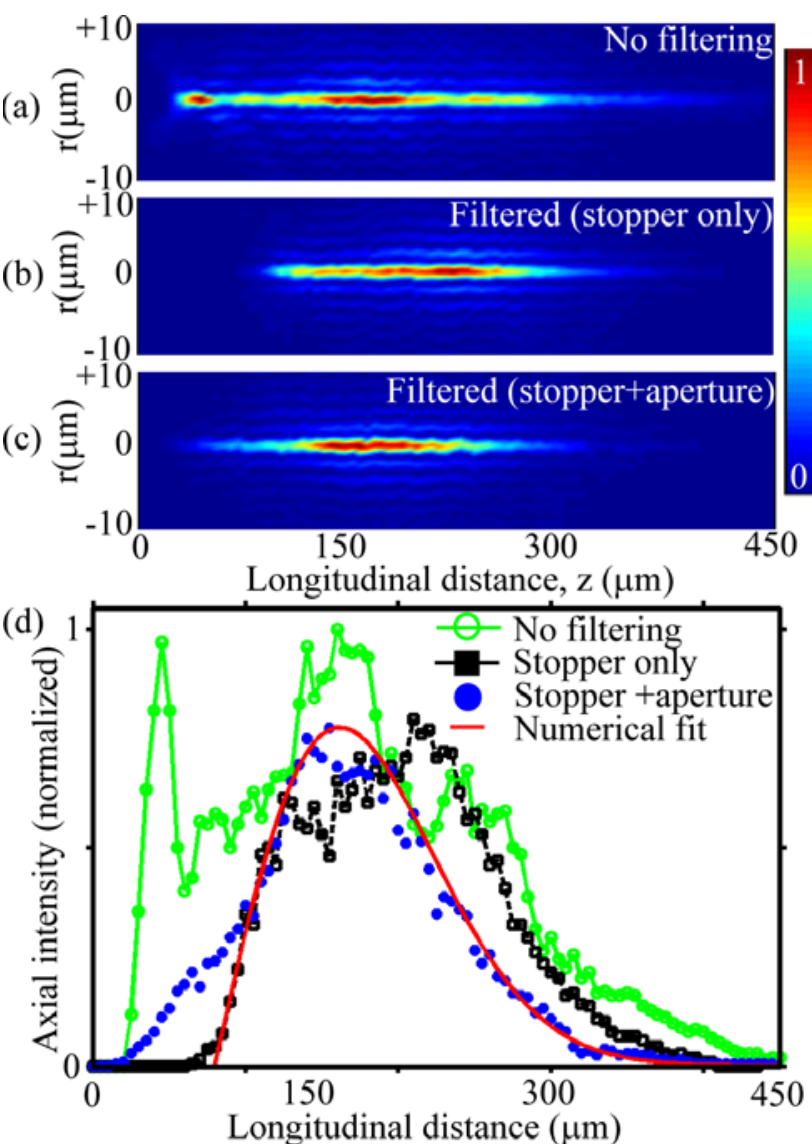

Fig. 2 Longitudinal cross-section profiles of experimentally generated zero-order Bessel beams $\left(\theta=12^{\circ}\right.$ in air) subjected to various filtering conditions. (a) Unfiltered Bessel beams. (b) Bessel beams filtered only with stopper. (c) High quality Bessel beams produced by filtering with both stopper and aperture. (d) Axial intensity distributions of Bessel beams subjected to different filtering schemes along with the axial profile of an ideal Bessel beam.

Under paraxial approximation, the diameter, D of the intense ring at the focal plane of lens L1 (i.e. diameter of the annular opening of spatial filter) can be estimated [19] as follows: $\mathrm{D}=2 \mathrm{f} \tan \left[\alpha\left(\mathrm{n}_{\mathrm{ax}}-1\right)\right]$. Considering the present experimental setting involving axicon $\left(\alpha=0.5^{\circ}\right.$, and $\mathrm{n}_{\mathrm{ax}}=$ 1.45), and lens L1 ( $\mathrm{f} 1=1000 \mathrm{~mm}$ ), $\mathrm{D}$ turns out to be 7.85 $\mathrm{mm}$. The estimated value of $\mathrm{D}$ is close to our experimental- 
ly measured value of $7.6 \mathrm{~mm}$. The whole assembly of spatial filter was placed at the focal plane of lens L1.

To characterize the generated Bessel beams, an imaging system consisting of an objective lens (Mitutoyo 20x / 0.42 NA), a plano-convex lens of focal length of $300 \mathrm{~mm}$ and a CCD camera (Thorlabs Inc., DCC1545M model) was employed. The transverse cross-sectional images of Bessel beams were recorded at each $5 \mu \mathrm{m}$ distance along the beam propagation direction. A 3D view of Bessel beams was reconstructed from the recorded transverse slices. Here, we show the longitudinal cross-sections of reconstructed Bessel beams subjected to different filtering conditions: (i) no filtering (see Fig. 2(a)), (ii) filtered with stopper only (see Fig. 2(b)) and (iii) filtered with both stopper and aperture (see Fig. 2(c)). The axial intensity profiles of all 3 generated Bessel beams along with an ideal Bessel beam are also shown in Fig. 2(d) illustrating how the axial intensity modulation was substantially removed using our proposed filtering system. The numerical fitting (see red line of Fig. 2 (d)) is based on the axial intensity profile of an ideal Bessel beam [20] as follows: $\mathrm{I}(\mathrm{z})=8 \pi \mathrm{P}_{0} \mathrm{z} \sin ^{2} \theta / \lambda \mathrm{w}^{2}$. exp [-2 $(\mathrm{z} \sin \theta / \mathrm{w})^{2}$, where $\mathrm{I}(\mathrm{z})$ is the peak intensity at a fixed position, $z$ along the beam propagation direction, $P_{0}$ is the peak power of the Gaussian laser beam incidents on the axicon, $\theta$ is the conical half-angle of the Bessel beam. $\lambda$ and $w$ are the wavelength and waist of the incident Gaussian laser beam respectively. For useful comparison, the intensity profiles were normalized for all the considered Bessel beams as shown in Fig. 2.

Using lens L2 (f2 = $20 \mathrm{~mm}$ ), the characteristics of resultant high-quality Bessel beams in air are as follows: conical half-angle $(\theta)$ of $12^{\circ}$, longitudinal extension $\left(Z_{N D}\right)$ of 130 $\mu \mathrm{m}$ FWHM and central core size (r) of $1.4 \mu \mathrm{m}$ FWHM. Following the same approach and lens L2 (f2 = $10 \mathrm{~mm}$ ), high angle Bessel beams were generated with following characteristics: $\theta=22^{\circ}, \mathrm{Z}_{\mathrm{ND}}=85 \mu \mathrm{m}$ FWHM and $\mathrm{r}=750$ nm FWHM.

\subsection{Bessel beam machining}

The generated high-quality Bessel beams were employed for single-shot machining of fused silica glass (a$\mathrm{SiO}_{2}$, refractive index, $\mathrm{n}=1.45$ ) samples of dimensions: length $\times$ breadth $\times$ height: $20 \times 10 \times 5 \mathrm{~mm}^{3}$. Note that when Bessel beams propagate in a medium of refractive index " $n$ ", their longitudinal extension gets lengthened by a factor of n. However, the Bessel central core size does not change with medium. Therefore, the estimated Bessel beam parameters in glass are as follows: (i) when L2 (f2 $=20$ $\mathrm{mm}$ ) was considered, $\theta=8.3^{\circ}$ (moderate focusing), $\mathrm{Z}_{\mathrm{ND}}=$ $190 \mu \mathrm{m}$ FWHM and $\mathrm{r}=1.4 \mu \mathrm{m}$ FWHM, (ii) when L2 (f2 = $10 \mathrm{~mm}$ ) was considered, $\theta=15^{\circ}$ (tight focusing), $\mathrm{Z}_{\mathrm{ND}}=$ $130 \mu \mathrm{m}$ FWHM and $\mathrm{r}=750$ nm FWHM.

The laser pulse energy was varied using neutral density filters. The laser pulse duration $\left(\tau_{\mathrm{p}}\right)$ was also observed to be an important parameter for Bessel beam machining as will be discussed in the following section. Therefore, the pulse durations were varied from tens of femtoseconds ( $\mathrm{fs}$ ) to few picoseconds (ps) by detuning the laser compressor i.e. manipulating the dispersion parameters by changing the distance between grating pairs of compressor system.
There are two modes of Bessel beam machining. (i) Bulk machining: The Bessel beam was suitably formed inside the glass mounted on a $\mathrm{X}-\mathrm{Y}-\mathrm{Z}$ motorized translation stage assembly. The resultant modified structures inside glass were primarily characterized using optical microscopy techniques. (ii) Surface machining: In this case, Bessel beam was formed in the glass in such a way that the resultant modified structures open up on its surface. Such methodology was followed to characterize the transverse dimensions of machined structures using both optical and scanning electron microscopy (SEM) techniques.

\subsection{Time-resolved microscopy imaging}

The Bessel pulse induced material excitation and relaxation processes in glass were studied over a temporal domain ranging from a few hundred femtoseconds to a few tens of microseconds using pump-probe microscopy techniques. In general, Bessel pulses (pump) at $800 \mathrm{~nm}$ wavelength and with pulse durations in the range of $50 \mathrm{fs}-6 \mathrm{ps}$, were used for material excitation and the dynamic characteristics of material after excitation were studied using two different modes of imaging: (i) Ultrafast imaging: The probe pulses were the second harmonic $(400 \mathrm{~nm})$ of Gaussian pulses at fundamental wavelength. The temporal resolution of these measurements was therefore within few picoseconds. (ii) Low-coherence multiscale imaging: The probe pulses were derived from a low-coherence laser source based on random lasing effect [21] and were realized using Rhodamine B solution mixed with latex nanobeads excited with a frequency doubled Nd: YAG laser pulse (7 nanoseconds (ns)). The temporal resolution of these measurements was therefore tens of nanoseconds, considering also the time jitter towards synchronizing involved fs and ns laser systems.

Note that the selection of probe laser pulse was based on the spatial coherence of the illumination source i.e. lowspatial coherence pulse reduces the speckle density and hence produces high quality image. In ultrafast imaging mode, the probe was derived from the pump pulse which has inherently high-spatial coherence and hence produces large number of speckles. Therefore, several tens of individual images were recorded using ultrashort probe pulse to obtain a representative image via averaging. In contrast, in long timescale imaging mode, a nanosecond laser source electronically synchronized with the pump pulse, was transformed into a low-spatial coherence random laser source without affecting its temporal duration to act as probe. This illumination arrangement therefore enabled us to capture single shot images of excited matrix until few tens of microseconds after laser excitation.

The imaging system was based on an upright optical microscope (Olympus BX-51) operating in both transmission (OTM) and phase contrast (PCM) modes. The probe pulse illuminates the Bessel pulse excited regions of glass in perpendicular geometry in both imaging modes. The images of the excited regions were thus recorded using EMCCD camera (ANDOR iXon Ultra 897) coupled with a suitable magnification system comprising of a tube lens and objective lenses i.e. when objective lens of 0.55 NA was used, the spatial resolution was $650 \mathrm{~nm}$. The OTM and PCM imaging modes were employed to track the absorp- 
tion and relative phase change of excited matrix respectively. Note that an object that scatters or absorbs light appears black in OTM and white in PCM mode. Also, the positive and negative phase change with respect to the background appears black and white in PCM mode respectively.

The number density of Bessel pulse induced carriers in glass was then estimated from the absorption characteristics of excited regions (derived from OTM images) at a fixed time delay and basing on the Drude model [22]. Scattering issues from small objects and other parameters were discussed in Ref. [22].

\section{Experimental results}

The results of single-shot machining and the carrier and structural dynamics corresponding to Type I and II material modifications are discussed in this section. We emphasize the role of pulse energy and temporal duration in controlling the nature of modification.

\subsection{Energetic conditions}

The pulse duration dependent morphological characteristics of Bessel beam $\left(\theta=8.3^{\circ}\right.$ in glass) induced material modifications in glass is illustrated in Fig. 3 corresponding to 2 different input pulse energies, $\mathrm{E}=4.1$ and $9 \mu \mathrm{J}$. Here, two perspectives can be seen.

From a technical viewpoint, in the case of ultrashort pulse duration i.e. in femtosecond range of pulse duration, Type I material modifications were obtained. As the pulse duration increases, the morphologies of material modifications gradually change from Type I to II. Indeed, high quality, long nanovoids (type II structures) were obtained in the case of machining using picosecond Bessel pulses. Here, "high quality" refers to uniform axial morphology with no discontinuous region. More importantly, the material modification was considered to be a void only when that appears bright in PCM and dark in OTM imaging modes [14].

From a fundamental viewpoint, beam propagation stability and energy deposition efficiency of nonlinear Bessel beams as a function of laser pulse duration were clearly apparent from Fig. 3; when the pulse energy is high, nonlinear phenomenon such as self-focusing (a power dependent process) is more pronounced, causing axially modulated plasma density inside the medium. Furthermore, the plasma defocusing effect is also strong for high energy cases, that limits the plasma density and hence the energy deposition along the Bessel central axis. Fig. 3 clearly elucidate such behavior in terms of strength and uniformity in axially deposited energy distribution. For instance, in the low energy case (i.e. $E=4.1 \mu \mathrm{J} /$ pulse), the strength of energy deposition is weak, resulting into Type I structures over the pulse duration range of $60 \mathrm{fs}-1.7 \mathrm{ps}$ as evident from Fig. 3(a). However, for $\tau_{p} \geq 2.6 \mathrm{ps}$, high quality voids were obtained, clearly advocating high level of energy deposition uniformly over the Bessel central axis. In contrast, due to the strong influence of above-mentioned nonlinear phenomena causing non-uniform energy deposition, axial uniformity of machined structures is compromised in the high energy case (i.e. $\mathrm{E}=9 \mu \mathrm{J} /$ pulse). As evident from Fig. 3(b), the structure machined with a laser pulse of $\tau_{p}=$ 1.7 ps, is a mixture of Type I and Type II zones. At this pulse energy, high quality voids were obtained only when laser pulses of $\tau_{\mathrm{p}} \geq 3.7$ ps were used. These results nevertheless indicate that an optimum balance between pulse energy and temporal duration is highly required for obtaining structures of desired choices.

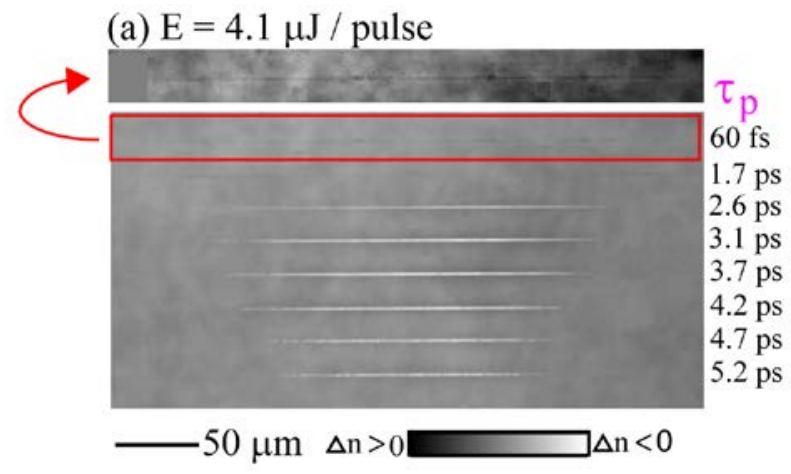

(b) $\mathrm{E}=9 \mu \mathrm{J} /$ pulse

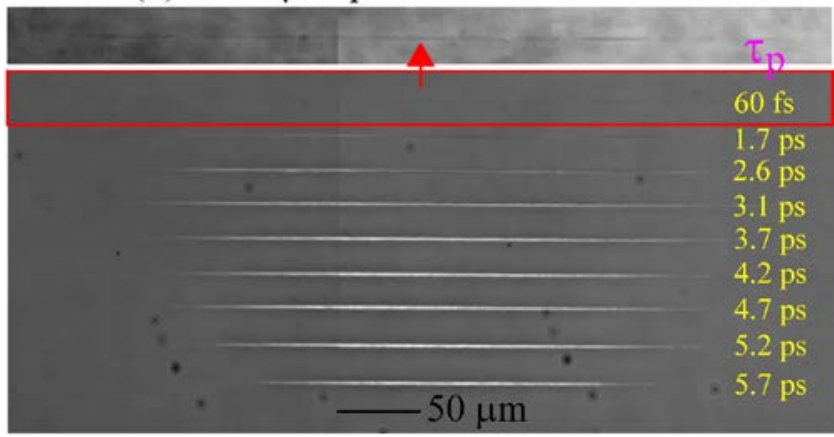

Fig. 3 Single-shot Bessel pulse $\left(\theta=8.3^{\circ}\right.$ in glass, moderate focusing) structuring results. Pulse duration dependent morphological characteristics of Bessel beam induced long structures are shown for 2 different pulse energies. (a) $E=4.1 \mu \mathrm{J}$ and (b) $E=9 \mu \mathrm{J}$. Insets to both (a) and (b) show the contrasted images of Type I structures machined using $60 \mathrm{fs}$ Bessel pulse. The color map of the relative index change is also given.

We identified the combinations of laser pulse energy and temporal duration towards fabricating high quality nanovoids in glass using zero-order Bessel beams as shown in Fig. 4(a). For a fixed laser pulse duration, pulse energies were varied and the morphologies of resultant machined structures were studied using optical microscopy. The blue and red data points of Fig. 4(a) represent the minimum and maximum pulse energies required to obtain high quality voids in glass respectively. The error bars represent the uncertainties with the pulse energy measurement. A SEM image (see Fig. 4(b)) showing typical void opening of 400 $\mathrm{nm}$ diameter machined in a stable void fabrication regime advocates the possibility of sub-wavelength size machining using Bessel pulse. Furthermore, by keeping the pulse duration fixed (i.e. $\tau_{\mathrm{p}}=4.7 \mathrm{ps}$ ), the dimensions and morphologies of Bessel pulse induced structures as a function of laser pulse energies were studied. Three different regimes were identified as illustrated in Fig. 4(c). The "onset" regime represents the evolutionary discontiguous structures. It is followed by, a "stable" regime with an energy range of $4.1<\mathrm{E}<18 \mu \mathrm{J} /$ pulse, where high quality nanovoids of lengths up to $400 \mu \mathrm{m}$ can be obtained. The diameters of such uniform nanovoids were measured to be in the range of $100-500 \mathrm{~nm}$ [14]. 

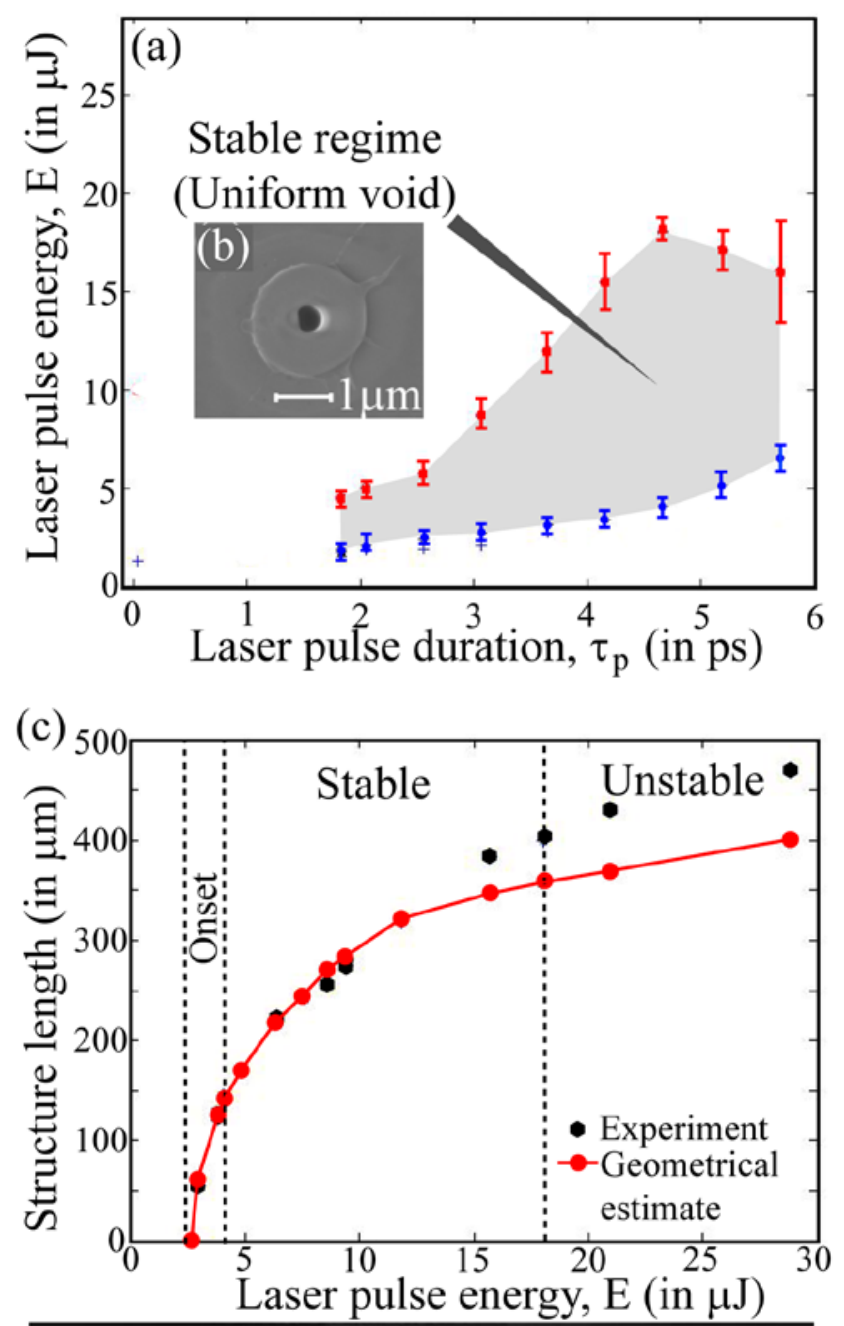

d) $50 \mu \mathrm{m}$

Fig. 4 (a) Parametric space in the framework of laser pulse energy and pulse duration for the fabrication of high quality voids inside glass in moderate focusing conditions. (b) SEM image of a void opening indicating sub-micron cross-section. (c) Variation of structure length with respect to laser pulse energy showing primarily the energetic conditions for uniform nanovoid fabrication using 4.7 ps Bessel pulse. (d) Typical optical micrograph of a non-uniform void fabricated using a high energy pulse. Red squared highlighted region represents the fragmented zone of such a void.

The lengths of nanostructures can also be theoretically estimated using a simple model [20] based on a pure geometrical approach, without considering any nonlinear effects. For a fixed input laser energy, the axial intensity, I(z) distribution (as discussed in Section 2.1) of corresponding Bessel beams was first retrieved. Then by assuming that the optical breakdown would happen inside glass only over the longitudinal zone exceeding a threshold intensity, $\mathrm{I}_{\text {th }}$ of 3.2 $\times 10^{12} \mathrm{~W} / \mathrm{cm}^{2}$, the breakdown zone length was assigned to the length of the resultant structure. Interestingly, the theoretically estimated structure lengths fairly match with the experimentally measured values as illustrated in Fig. 4(c). As the pulse energy was increased further, material modification with a strange undesired structural morphology was observed i.e. fragmented region at the center of void as shown in Fig. 4(d). Such a behavior was observed to be common for picosecond Bessel pulse machining involving high energy pulses and the corresponding fabrication regime is termed as "unstable” regime.

\subsection{Ultrafast dynamics}

In order to understand how silica glass behave under Bessel pulse excitation, the relevant carrier and structural dynamics on ultrafast timescale (i.e. few hundred femtoseconds to $17.5 \mathrm{~ns}$ ) were first studied. Fig. 5 shows carrier density evolution and relaxation characteristics corresponding to both Type I and II structures produced by Bessel beams of $8.3^{\circ}$ in glass. The experimental and calculation details are given in Ref. [22].

In the case of femtosecond Bessel laser pulse (60 fs, 7 $\mu \mathrm{J}$ energy) excitation of glass leading to Type I structure, the carrier number density, $\mathrm{N}_{\mathrm{e}}$ swiftly increases reaching a maximum value i.e. about an order of magnitude less than its critical value in vacuum $\left(\mathrm{N}_{\mathrm{cr}}=1.74 \times 10^{21} / \mathrm{cm}^{3}\right.$ for 800 $\mathrm{nm}$ light) and decreases to almost zero within few picoseconds after laser excitation as shown in Fig. 5(a). This behavior is often associated with carrier trapping [22] (with a life time of 150 fs in $\mathrm{SiO}_{2}$ matrix [23]). Such a phenomenon assists self-trapped exciton formation which upon relaxation decays into electronic defects known as E' and non- bridging oxygen-hole center (NBOHC). Here, NBOHC defects resulting from bond-breaking are assumed to correlate to the formation of smaller $\mathrm{Si}-\mathrm{O}$ rings and hence to the creation of more compact matrix. This results in an increased refractive index structure (Type I) as shown in Fig. 5(a) (top). In contrast, the relaxation characteristics of glass excited with a picosecond Bessel laser pulse are different than the femtosecond laser pulse excitation.

Fig. 5(b) shows the relevant carrier dynamics of a Type II void-like structure obtained with a picosecond Bessel pulse (4.7 ps, $7 \mu \mathrm{J}$ energy). In this case, the carrier number density grew up to near-critical value and almost maintained its strength until 17.5 ns, without showing clearly observable signature of carrier trapping. Besides, pressure waves were visualized beyond 1 ns after laser excitation. The insets to Fig. 5(b) show PCM images of the Bessel excited regions and the emergence of travelling pressure waves at $1.5 \mathrm{~ns}$ and $6.25 \mathrm{~ns}$ delay positions. Earlier studies on light-matter interaction involving Gaussian laser beams and transparent materials have also revealed such material relaxation characteristics by observing pressure waves on nanosecond time scales [24-26]. This indicates the onset of thermo-mechanical phenomena. The above observations clearly indicate high level excitation of matter leading to a high temperature and pressure state. As a consequence, a microexplosion might have occurred, compressing the material along outward direction and leaving behind a rarified zone at the excitation epicenter. This scenario would involve a sufficient pressure rise to overcome the mechanical resistance of glass in the range of few GPa [4]. The existence of high temperature and pressure state is also evidenced by the SEM image (see inset of Fig. 5(b)) showing the opening of a channel produced using surface machining technique and by maintaining other experimental conditions same as that of bulk machining. The spread of molten material around the channel indicates that the hot excited matter must have ejected from the bulk and deposited around the channel. Considering all the provided arguments, 
it is likely to correlate the void-like structure formation to high temperature and pressure driven thermo-mechanical evolution of glass [22]. However, the dynamics of this process and the related timescale are important for defining the actual thermo-mechanical path. This motivated us to study further the long timescale dynamics of void-like material modification [27]
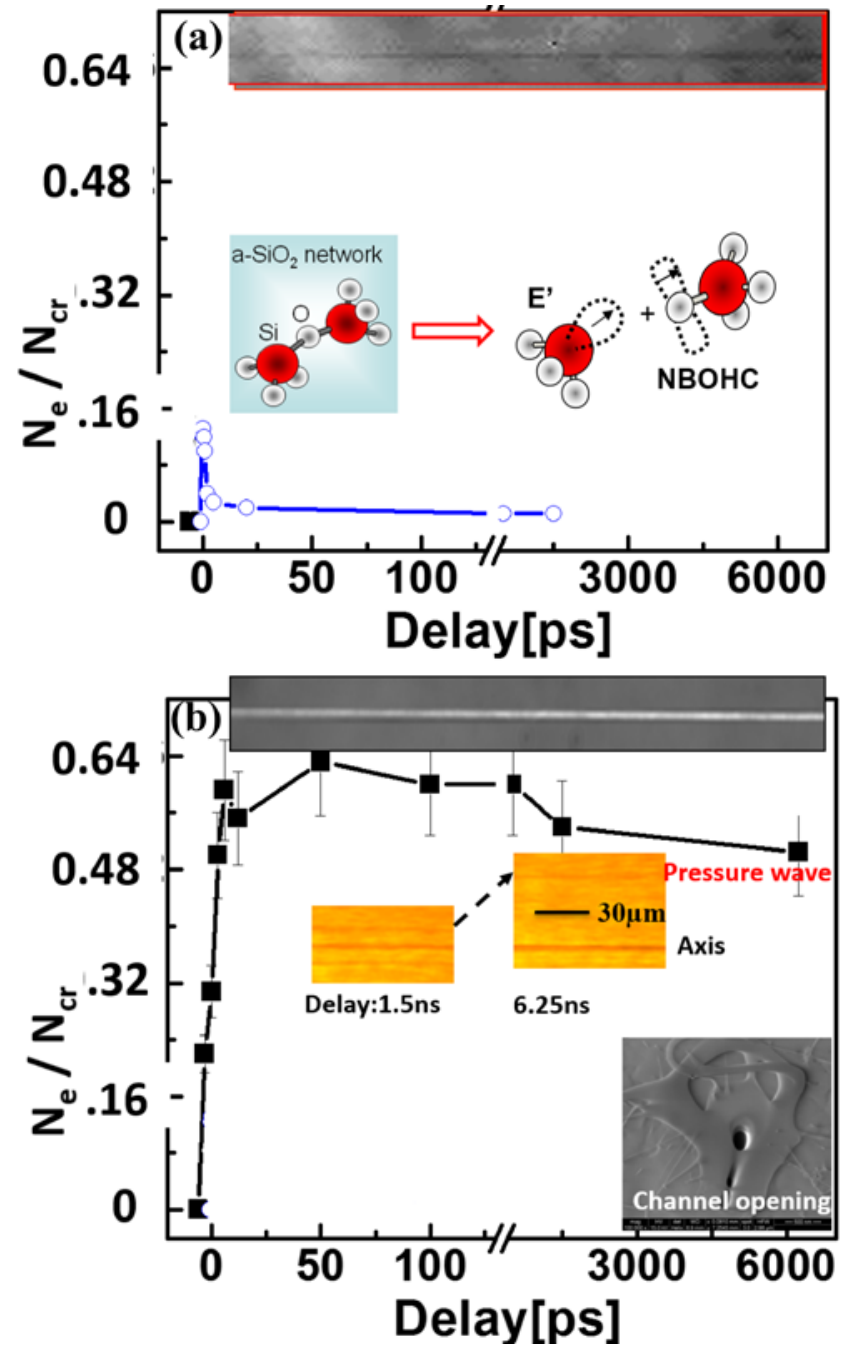

Fig. 5 Ultrafast dynamics of Bessel pulse $\left(\theta=8.3^{\circ}, 7 \mu \mathrm{J}\right.$ energy) induced structures inside glass at two different pulse durations resulting in Type I and II structures. Variation of carrier density with respect to delay time (after laser excitation) in the cases of a femtosecond laser (60 fs) induced Type I structure (a) and picosecond laser (4.7 ps) induced Type II structure such as void (b). Typical optical micrographs corresponding to both the cases are shown as insets to both (a) and (b), alongside with the emergence of pressure waves visualized at ns delays in PCM in (b).

\subsection{Long timescale structural dynamics}

A multiscale observation is important to cover the whole matter relaxation cycle as discussed in Ref. [27]. This study deals with the evolutionary characteristics of glass excited by a femtosecond Bessel pulse $\left(\theta=15^{\circ}, 1.8\right.$ $\mu \mathrm{J}$ energy) in tight focusing conditions. The random laser probe pulse illuminates the Bessel excited region and snapshots (in PCM mode) were recorded over a broad temporal range i.e. $1 \mathrm{~ns}-11 \mu \mathrm{s}$ as shown in Fig. 6. Note that the image recorded at $11 \mu$ s delay time is similar to that of a permanent modification, which indicates that the excited matter has indeed stabilized at this time scale.

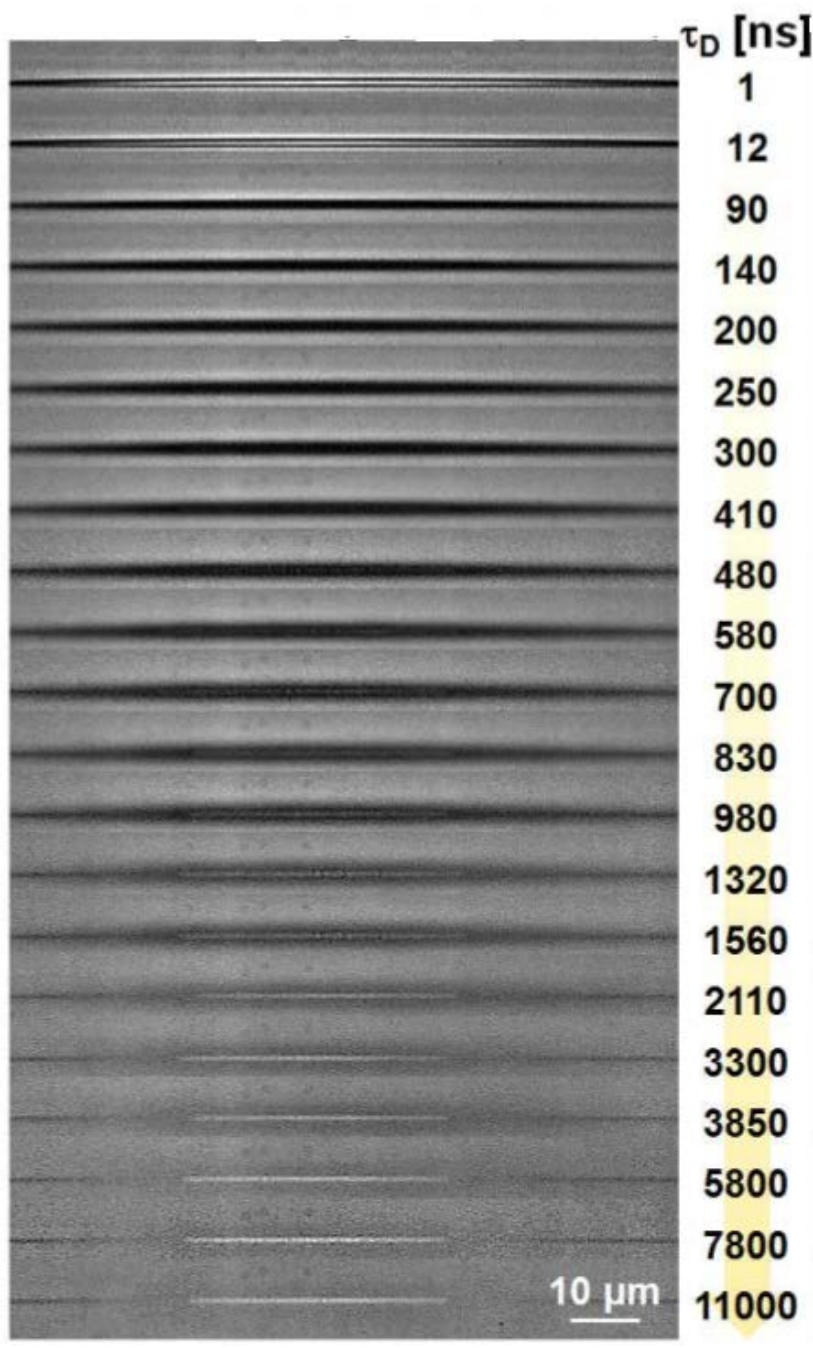

Fig. 6 Long timescale structural dynamics of Bessel pulse $(\theta=$ $15^{\circ}, \mathrm{E}=1.8 \mu \mathrm{J}, \tau_{\mathrm{p}}=50 \mathrm{fs}$ ) excited regions of silica glass. PCM images of excited regions corresponding to various time delays (after laser excitation) in the ns- $\mu$ s range are shown. Note that the structure corresponding to $11 \mu$ s represents the final modified material.

Several observations can be made from Fig. 6. First, white regions at the center of images (excitation profiles) existed for first few tens of nanoseconds. We independently verified through photoluminescence measurements that the carrier plasma (identified as such based on the correlations between luminescence and phase contrast measurements) stays live until few tens of nanoseconds in such fabrication conditions and appears white in our snapshot images. Followed by, excitation profiles completely shaded with dark regions, representing dense or hot phase of matter starts to appear and existed until few hundred nanoseconds after laser excitation. This is identified as a hot low viscosity (liquid) phase. As the time increased i.e. beyond 500 ns, a white region at the center of excited zone starts to appear, which we assign to void-like phase of glass. The laterally-evolving shadow-like regions indicate the heat transport (via a transient positive index change associated with the thermo-optic effect). 
Note that the timescale (few hundred nanoseconds) for the emergence of void-like phase of matter in our experiments is very different from the earlier reported [4] shockrarefaction assisted void formation (in few picoseconds), perhaps in the view of the local estimated pressure (few GPa of electronic pressure in the present case [27]). Based on the slow dynamics, we attribute the void opening to the cavitation phenomenon in high temperature and pressure liquid phase of glass. The cavitation evolves towards the emergence of void in the cooling phase [27]. The laser induced stress can further drive the material rupture. It is not yet clear if mechanical fracture may equally take place on a longer run. Thus, our experimental findings on liquid cavitation / thermo-mechanical relaxation based void fabrication opens novel opportunities of material synthesis [4, 28] and give a characteristic timescale to the process of nanostructuring.

\section{Conclusions}

The Bessel pulse excitation of fused silica glass was studied in the framework of ultrafast laser nanofabrication. Material modifications in terms of both increased and decreased refractive index were obtained inside glass on single-shot basis. Most importantly, we have discovered that for a fixed cone angled Bessel beam, long uniform voids can be fabricated over a finite range of laser pulse energy corresponding to a particular laser pulse duration. For instance, extended voids of sub-500 nm diameters and lengths up to $400 \mu \mathrm{m}$, were machined in glass using picosecond Bessel pulses. The carrier and structural relaxation dynamics associated with Bessel pulse induced nanostructures, over a temporal domain ranging from subpicoseconds to few tens of microseconds were also uncovered. The electronic defect-assisted material compaction is held responsible for the formation of increased refractive index structure. In contrast, high temperature and pressure driven liquid cavitation accompanied by stress relaxation on a rather slow time scale i.e. few hundred nanoseconds after excitation are held responsible for the formation of decreased refractive index structure like void.

\section{Acknowledgments}

We thank A. Soleihac and R. Antoine for their support in the experimental setup and random laser illumination. We also acknowledge Agence Nationale de la Recherche (Project: ANR 2011 BS04010 NanoFlam and ANR 2011 BS09026 SmartLasir) for the financial support. LABEX MANUTECH-SISE (ANR-10-LABX-0075) of the Université de Lyon, within the program "Investissements d'Avenir" (ANR-11-IDEX-0007) operated by ANR is also acknowledged for the financial support.

\section{References}

[1] K. Itoh, W. Watanabe, S. Nolte, and C. B. Schaffer: MRS Bulletin, 31, (2006) 620.

[2] R. R. Gattass, and E. Mazur: Nat. Photonics, 2, (2008) 219.

[3] K. Sugioka, and Y. Cheng: Light Sci. Appl., 3, (2014) e149.
[4] S. Juodkazis, K. Nishimura, S. Tanaka, H. Misawa, E. G. Gamaly, B. Luther-Davies, L. Hallo, P. Nicolai, and V. T. Tikhonchuk: Phys. Rev. Lett., 96, (2006) 166101.

[5] S. I. Kudryashov, G. Mourou, A. Joglekar, J. F. Herbstman, and A. J. Hunt: Appl. Phys. Lett., 91, (2007) 141111.

[6] J. F. Herbstman, and A. J. Hunt: Opt. Express, 18, (2010) 16840.

[7] J. Durnin, J. J. Miceli and J. Eberly: Phys. Rev. Lett., 58, (1987) 1499.

[8] D. McGloin, and K. Dholakia: Contemp. Phys., 46, (2005) 15.

[9] F. Courvoisier, R. Stoian, and A. Couairon: Opt. Laser Tech., 80, (2016) 125.

[10]A. Marcinkevičius, S. Juodkazis, S. Matsuo, V. Mizeikis, and H. Misawa: Jpn J. Appl. Phys., 40, (2001) L1197.

[11] J. Amako, D. Sawaki, and E. Fujii: J. Opt. Soc. Am. B 20, (2003) 2562.

[12] Y. Matsuoka, Y. Kizuka, and T. Inoue: Appl. Phys. A, 84, (2006) 423.

[13]M. K. Bhuyan, F. Courvoisier, H. S. Phing, O. Jedrkiewcz, S. Recchia, P. Di Trapani, and J. M. Dudley: Eur. Phys. J. Special Topics, 199, (2011) 101.

[14] M. K. Bhuyan, P. K. Velpula, J. P. Colombier, T. Olivier, N. Faure, and R. Stoian: Appl. Phys. Lett., 104, (2014) 021107.

[15] W. J. Tsai, C. J. Gu, C. W. Cheng, and J. B. Horng: Opt. Eng., 53, (2013) 051503.

[16] M. K. Bhuyan, O. Jedrkiewicz, V. Sabonis, M. Mikutis S. Recchia, A. Aprea, M. Bollani, and P. Di Trapani: Appl. Phys. A, 120 (2015) 443.

[17] J. H. McLeod: J. Opt. Soc. Am., 44, (1954) 592.

[18] O. Brzobohaty, T. Cizmar, and P. Zemanek: Opt. Express, 16, (2008) 12688.

[19] M. Rioux, R.Tremblay, and P. A. Belanger: Appl. Opt., 17, (1978) 1532.

[20] V. Jarutis, R. Paskauskas, and A. Stabinis: Opt. Commun., 184, (2000) 105.

[21] A. Mermillod-Blondin, H. Mentzel, and A. Rosenfeld: Opt. Lett., 38, (2013) 4112.

[22]P. K. Velpula, M. K. Bhuyan, F. Courvoisier, H. Zhang, J. P. Colombier, and R. Stoian: Las. Photon. Rev., 10, (2016) 230.

[23] S. S. Mao, F. Quere, S. Guizard, X. Mao, R. E. Russo, G. Petite, and P. Martin: Appl. Phys. A, 79, (2004) 1695.

[24] M. Sakakura, and M. Terazima: Phys. Rev. B, 71, (2005) 024113.

[25] A. M. Blondin, J. Bonse, A. Rosenfeld, I. V. Hertel, Y. P. Meshcheryakov, N. M. Bulgakova, E. Audouard, and R. Stoian: Appl. Phys. Lett., 94, (2009) 041911.

[26] Y. Hayasaki, M. Isaka, A. Takita, and S. Juodkazis: Opt. Exp., 19, (2011) 5725.

[27] M. K. Bhuyan, M. Somayaji, A. Mermillod-Blondin, F. Bourquard, J. P. Colombier, and R. Stoian: Optica, 4, (2017) 951.

[28] L. Rapp, B. Haberl, C. J. Pickard, J. E. Bradby, E. G. Gamaly, J. S. Williams, and A. V. Rode: Nat. Commun., 6, (2015) 7555. 